\title{
PERCEPCIÓN ACERCA DEL CÁNCER DE MAMA EN UN GRUPO DE MUJERES DE UN HOSPITAL EN POPAYÁN, COLOMBIA
}

\section{BREAST CANCER PERCEPTION IN A GROUP OF WOMEN IN A HOSPITAL IN POPAYÁN, COLOMBIA}

\author{
Nancy Janneth Molano-Tobar ${ }^{1}$ \\ Patricia Eugenia Vélez Varela \\ Universidad del Cauca, Colombia \\ najamoto@unicauca.edu.co
}

\begin{abstract}
Resumen
El objetivo de este artículo fue identificar la percepción del cáncer de mama que tienen un grupo de mujeres en la Ciudad de Popayán, Colombia. Metodología. Se realizó un estudio con enfoque cualitativo, en el cual se aplicaron técnicas como la observación y la entrevista a profundidad con cuestionarios semiestructurados, grabaciones en audio y bitácoras. El trabajo de campo se realizó entre el I y II periodo de 2015, con 39 pacientes mujeres con edad promedio de 46,70 años, que tuvieron como diagnóstico cáncer de mama estadio II y que asisten al Hospital Universitario San José, de la Ciudad de Popayán, Colombia. Resultados. Se obtuvieron cuatro categorías: cáncer una enfermedad heredada; angustia y depresión por el futuro; insatisfacción corporal y sexual; y la familia un apoyo fundamental. Conclusiones. El cáncer de mama es una enfermedad de alto riesgo, tanto económico como social, desde la visión de salud pública. En el ámbito personal, genera en las mujeres sentimientos de angustia y depresión los cuales conllevan a duda por su futuro y temor a heredar la enfermedad. Una particularidad manifestada es la inseguridad e insatisfacción corporal que está afectando sus relaciones sociales y de pareja, propiciando en la mujer un deterioro en su autoestima, autoconfianza y vulnerabilidad emocional, lo que incide en la calidad de vida de la población. Por ello, es importante un equipo interdisciplinario que respalde las diferentes situaciones que afrenta la mujer y su familia.
\end{abstract}

Palabras claves: cáncer, mujer, salud, depresión, familia

\begin{abstract}
The objective of this paper was to identify the perception of breast cancer in a group of women in the city of Popayán, Colombia. Methodology. A qualitative study was conducted, applying techniques such as observation and in-depth interviews with semi-structured questionnaires, audio recordings and logs. Fieldwork was conducted between periods I and II of 2015. A total of 39 female patients participated with an average age of 46.70 years, who were diagnosed with stage II breast cancer and were being treated at Hospital Universitario San José, City of Popayan, Colombia. Results. Four categories were obtained: cancer as an inherited disease; anxiety and depression for the future; physical and sexual dissatisfaction; and family as essential support. Conclusions. From the public health perspective, breast cancer is a high-risk disease, both economically and socially. From an individual perspective, it generates feelings of anxiety and depression in women, leading to doubts about their future and fear to pass it to their children. A particularity that manifested was body insecurity and
\end{abstract}


dissatisfaction, which affects their social and romantic relationships, deteriorates their self-esteem, self-confidence and emotional vulnerability and affects the quality of life of this population. Consequently, it is important to have an interdisciplinary team to support the different situations faced by women and their families.

Keywords: Cancer, Women, Health, Depression, Family

\section{Introducción}

La salud, para el ser humano, es un factor de suma importancia y debe abordarse de manera integral. En coherencia con ello, "salud se refiere a la satisfacción de un individuo con los aspectos físicos, sociales y psicológicos de su vida" (Varela, Arrivillaga, Cáceres, Correa Sánchez y Holguin, 2005, p. 72), lo que permite indicar que "los cambios en el estado de salud responden a una multicausalidad y a los factores que intervienen en esta, se los conoce como determinantes de la salud" (Londoño y Chaparro, 2011 p. 15).

Sabiendo que el cáncer en la actualidad es uno de los procesos patológicos que genera grandes consecuencias en la persona y familia, se considera que "el cáncer de mama (CaMa) es un problema de salud pública no reconocido como prioritario por la mayoría de las autoridades de salud en la región de las Américas, pese al continuo incremento de las tasas de incidencia y mortalidad” (González, González, Nigenda y López, 2010, p. 533).

El nombre de cáncer "deriva de la palabra kankros, que significa cangrejo y hace referencia a la similitud en que el animal y la enfermedad "atrapan" y destrozan a sus víctimas" (Figueredo, 2008, p. 1). En palabras de Palacios y Castaño (2013, p. 505), "el cáncer se representa entonces como un personaje vivo con características casi humanas; se transforma, se camufla, es implacable, voraz, un intruso capaz de destruir la vida de una persona", ya que no solo afecta la dimensión física, sino que también involucra la esfera psicológica y social.

El cáncer es la neoplasia maligna más estudiada en el mundo, aproximadamente "1,7 millones de mujeres fueron diagnosticas con esta enfermedad en el 2012" (Chen, Wen, Li, Luo y Zhang, 2016, p. 12), y "se prevé que para el 2020 se presentarán cerca de 15 millones de casos nuevos por año" (Guevara, 2007, p. 64), por lo que se considera como una prioridad a nivel mundial. Este proceso neoplásico "se presenta con mayor frecuencia a partir de los 45 años de edad, periodo que coincide con la aparición de la menopausia” (Valle et al., 2006 p. 328), lo anterior se justifica debido a que después de la menopausia, "los tejidos como el parénquima mamario, son muy sensibles a los estrógenos, quedando expuestos a un mayor estimulo" (Aguilar, González, García, Álvarez y Padilla, 2012, p. 900), conllevando a un riesgo de desarrollar cáncer.

La etiología de la enfermedad es conocida parcialmente, ya que se han encontrado diferentes factores que combinados aumentan el riesgo. Además, se ha identificado que el cáncer de mama se debe "en parte, a factores epigenéticos como las características ambientales, socioeconómicas y de estilo de vida" (Pou et al., 2014, p. 619), sin dejar de lado los factores 
genéticos asociados a "genes de alta como de baja penetración" (Sanabria, Muñoz y Vargas, 2009, p. 62). Asimismo, diversos estudios plantean una fuerte asociación con la herencia, factores "de los cuales el 5\% se puede asociar con mutaciones en genes de alta penetrancia como BRCA1 y BRCA2” (Pinto, Sánchez, Ibañez y Ramírez, 2006, p. 7). Por otra parte, "se ha documentado que la edad temprana a la menarquia, la nuliparidad o la edad tardía al primer embarazo, la falta de lactancia y la edad tardía de la menopausia" (Knaul et al., 2009, p. 138) pueden ser otras causas probables que, combinadas con hábitos y estilos de vida inadecuados, son coadyuvantes a la presencia del cáncer de mama.

Es indudable que cuando la mujer tiene un diagnóstico de cáncer, la percepción de su mundo se altera, afectando la estabilidad psicológica del individuo, encontrando que el cáncer no solo afecta la estructura física de la persona que lo padece, sino que también tiene repercusiones que "se expresan en todas las esferas de la vida: la familiar, la relación de pareja y la laboral y social" (García y González, 2007, p. 73). Estudios de Palacios, Gonzales y Zani (2015, p. 498) evidencian cómo el impacto del cáncer afecta "las esferas afectiva, emocional, cognitiva y comportamental en el paciente con cáncer y en su familia”, con lo que se demuestra que no es solo una afección orgánica sino que abarca otras dimensiones. Sumado a ello, dicha autopercepción varia "dependiendo de la severidad del proceso de la enfermedad, del grado y tipo de incapacidad” (Baider, 2003, p. 507).

Debido a esto, es importante identificar el sentir de las mujeres con cáncer de mama acerca de su enfermedad y así brindar pautas para un tratamiento más incluyente, ya que "para la mujer, la aparición de un tumor maligno en los órganos sexuales constituye un impacto psicológico capaz de desestructurar el equilibrio anímico y afectivo" (Bejarano, Fuchs, Fernández y Amancio, 2009 , p. 183), pues "en el caso de la mujer, las mamas han constituido generalmente una parte importante de la belleza corporal, un símbolo de la sexualidad y, de hecho, una zona erógena importante, así como una representación de la maternidad" (García y González, 2007, p.7 5). Por lo tanto, esta situación genera "sentimientos de ansiedad, tristeza, pérdida y miedo, así como dudas y cuestionamientos, debido al estigma de enfermedad terminal que ocasiona sufrimiento y muerte" (Mesquita, Magalhães, De Almeida, Carvalho y Mota, 2007, p. 2). Adicionalmente, en la mujer se desencadenan una serie de preguntas y manifestaciones psicológicas asociadas consigo misma, con su familia y con las expectativas al futuro.

Ante lo expuesto, se resalta la utilidad de conocer la percepción que las mujeres tienen sobre el cáncer de mama, para posteriormente plantear tratamientos desde una mirada holística. En este sentido, el presente trabajo es resultado de un proceso investigativo derivado del proyecto de Candidatura del Doctorado en Ciencias Biomédicas de la Universidad del Valle, Colombia, denominado "Factores Epigenéticos relacionados con la calidad de vida en mujeres del Sur-Occidente Colombiano”. Con este artículo se busca identificar la percepción del cáncer de mama que tienen las mujeres en la Ciudad de Popayán-Cauca, Colombia. 
Nancy Janneth Molano-Tobar

Patricia Eugenia Vélez Varela

URL: http://www.revistas.una.ac.cr/mhsalud

\section{Metodología}

\section{Participantes}

Las participantes de esta investigación fueron mujeres que asistieron a controles oncológicos en un Hospital Universitario San José en la Ciudad de Popayán, Colombia. La muestra se seleccionó por conveniencia y correspondió a 39 mujeres con un promedio de edad de 46,70 años $\pm 1,48$. Todas fueron diagnosticadas con cáncer de mama y fueron distribuidas, según la clasificación, en 12 mujeres con cáncer de mama tipo IIa (invasivos con ganglios axilares), 13 mujeres con cáncer IIb (invasivo con ganglios afectados) y 14 mujeres con cáncer de mama tipo invasivo en ganglios axilares y esternón. Se tuvieron en cuenta los siguientes criterios de inclusión: mujeres con diagnóstico de cáncer de mama de acuerdo al tipo descrito anteriormente, que asistieran regularmente tanto a los controles como a los procesos de rehabilitación, que decidieran por voluntad propia asistir constantemente a las reuniones y que aceptaran firmar el consentimiento informado, donde se les garantizó el manejo ético y anónimo. Para esto, se asignaron códigos de análisis regidos por los siguientes elementos: un número y la letra $\mathrm{R}$, de acuerdo con el número de relato, seguido por el número de la sesión en que se realizó.

\section{Materiales y métodos}

La metodología empleada para este trabajo fue cualitativa, con el fin de obtener un conocimiento a fondo acerca de lo que pensaban y sentían las mujeres con cáncer de mama. El diseño fue de tipo fenomenológico, en el que se explora, describe y comprende lo que los individuos tienen en común (Hernández, Fernández y Baptista, 2014). Las técnicas empleadas fueron la observación y la entrevista en profundidad, con cuestionarios semiestructurados, grabaciones en audio y bitácoras. El trabajo de campo se realizó entre el I y II periodo de 2015.

\section{Procedimiento y análisis estadístico}

Se basó en el enfoque fenomenológico hermenéutico propuesto por Hernández Sampieri et al. (2014). Este consistió en:

“a) definir un fenómeno o problema de investigación, que correspondió a mujeres con diagnóstico de cáncer de mama, en cualquier estadio; b) estudiarlo y reflexionar sobre este, se realizó búsqueda de antecedentes concernientes al tema de investigación y con el conocimiento teórico se realizaron encuentros con las mujeres, donde las entrevistas en profundidad y diarios de campo, permitieron identificar parámetros comunes, después de una relectura del material y clasificación de la información, en unidades de análisis; c) descubrir las categorías y temas esenciales del fenómeno, que fueron depuradas mediante 
las técnicas de triangulación obteniendo las categorías axiales, que dieron origen a la codificación selectiva desarrollando las ideas finales para d) describirlo, e) interpretarlo".

Para el procesamiento de la información suministrada y categorización se utilizó el programa MAXQDA versión 12.

\section{Aspectos ético-legales}

Se tuvo en cuenta lo dispuesto en la Declaración de Helsinki (1975) y los dispuesto en la Resolución 8430 del Ministerio de Salud y Proteccion Social (1993) de Colombia para investigación con personas. Al grupo de apoyo para la investigación se le ofrecieron charlas sobre los aspectos éticos y como debían asumirse los abordajes y encuentros. Además, se presentó el proyecto a las mujeres con cáncer de mama $\mathrm{y}$, una vez explicado y comprendido, se diligenció el formato de consentimiento informado, donde se garantiza la protección a la intimidad, a la confidencialidad y el derecho al anonimato tanto de los datos suministrados como de las entrevistas. Por lo tanto, este estudio se considera con riesgo mínimo de lesión para los participantes.

\section{Resultados}

Los resultados evidencian, como se puede ver en la tabla 1, parámetros cuantitativos que indican con mayor claridad los aspectos sociodemográficos.

Tabla 1.

Descriptivos generales del grupo de mujeres en estudio

\begin{tabular}{lll}
\hline & Media & Desviación estándar \\
\hline Edad & 46,70 & 1,48 \\
\hline Edad de aparición del CaMa & 31,8 & 3,78 \\
\hline
\end{tabular}

La siguiente tabla enuncia la frecuencia relacionando el estado civil de la población, en el que se encontró un alto porcentaje de mujeres con una pareja estable.

Tabla 2.

Frecuencia del estado civil del grupo de mujeres en estudio

\begin{tabular}{ccc}
\hline Estado civil & Frecuencia & Porcentaje \\
\hline Casado & 15,6 & 40,0 \\
\hline Soltero & 1,56 & 4,0 \\
\hline Divorciada & 14,04 & 36,0 \\
\hline Viuda & 7,8 & 20,0 \\
\hline Total & 39 & 100,0
\end{tabular}


URL: http://www.revistas.una.ac.cr/mhsalud

De la misma manera, se presenta la frecuencia relacionada con el estadio de la enfermedad en las participantes del estudio.

Tabla 3.

Distribución de los estadios del cáncer del grupo de mujeres en estudio

\begin{tabular}{lcc}
\hline \multicolumn{1}{c}{ Estadio } & Frecuencia & Porcentaje \\
\hline Cáncer invasivos con ganglios afectados & 13 & 33,3 \\
\hline Cáncer invasivo en ganglios axilares & 12 & 30,8 \\
\hline Cáncer invasivo en ganglios axilares y esternón & 14 & 35,9 \\
\hline Total & 39 & 100,0 \\
\hline
\end{tabular}

Uno de los reportes evidenciados en la población fue la alta frecuencia de familiares que han presentado cáncer. Estos datos se muestran en la siguiente tabla.

Tabla 4.

Distribución de antecedentes con cáncer del grupo de mujeres en estudio

\begin{tabular}{lcc}
\multicolumn{1}{c}{ Antecedentes familiares } & Frecuencia & Porcentaje \\
Ninguno & 5 & 12,8 \\
Solo un familiar & 5 & 12,8 \\
De 2 a 3 familiares & 18 & 46,2 \\
Más de 4 familiares & 11 & 28,2 \\
Total & 39 & 100,0
\end{tabular}

Las mujeres refieren que los antecedentes familiares de cáncer se manifiestan en diferentes órganos como pulmón (25\%), faringe (10\%) y colon (20\%), mientras que la mayor población con antecedentes prevaleció en glándula mamaria con un $35 \%$.

Del proceso investigativo desarrollado que se logró evidenciar cuatro categorías investigativas: i) el cáncer como una enfermedad heredada; ii) angustia y depresión por el futuro; iii) insatisfacción corporal y sexual; y iv) la familia es un apoyo fundamental.

Las entrevistas develaron que la mayoría de las mujeres provienen de familias con antecedentes de diversos cánceres. En este grupo, las mujeres enuncian que tanto sus madres como sus abuelas murieron por esta enfermedad, situación que para ellas genera un sello que no quieren continuarlo. Estos comentarios develaron la categoría "el cáncer como una enfermedad heredada", en la que se muestra cómo las mujeres presentan sentimientos de temor porque sus familiares puedan heredar esta enfermedad, de forma que sea más invasivo, más agresivo y con consecuencias mayores en sus hijas. Algunos de sus comentarios se citan a continuación. 
"He sufrido esta enfermedad con mis tías y ahora conmigo. Dios no quiera que esta lotería se la ganen mis hijas también" (6R8).

"Hablando con mi familia, hemos llegado a la conclusión que, desde mi abuela, mi mama y yo hemos desarrollado esta enfermedad, ahora se sabe y se puede hacer algo, pero antes ellas se murieron sin poder hacer nada, (...) ha sido una herencia que no se la quiero dejar a nadie" (34R16).

Aunque estudios previos demuestran que el cáncer en estadios tempranos no representa mayores alteraciones en la corporalidad de la mujer, en el grupo de trabajo se logró evidenciar que la incidencia del cáncer en un estado más avanzado genera cambios en su cuerpo y repercusiones en su sexualidad. Esto se evidencia ya que las mujeres dan cuenta de una disminución relacionada con la frecuencia en sus encuentros sexuales de pareja y también mencionan que en muchas situaciones se cohíben a relacionarse con otras personas fuera de su núcleo familiar inmediato, ya que el cambio en su apariencia física se sobrepone no solo al plano emocional, sino que también se asocia a su sexualidad. De esta manera, estas mujeres perciben el reflejo de otra persona que en realidad no son, de ahí que surgiera la categoría "insatisfacción corporal y sexual". Ejemplo de ello son los siguientes comentarios.

“(...) me veo al espejo y me siento castrada, deforme, ya no soy yo, no la misma, y ¿si no me veo bonita así? cómo voy a mostrarme delante de mi esposo, me da vergüenza de lo que el cáncer me ha dejado" (23R28).

"Con esta situación no le dan ganas a uno de nada, ni arreglarse, lo que uno ve en el espejo es a otra cosa, otro cuerpo" (15R4).

“(...) tengo relaciones muy pocas veces ya, no me dan ganas y lo hago ya por mi esposo, que por el deseo de estar con él” (18R25).

En un diagnóstico, la palabra cáncer se relaciona inmediatamente para las mujeres con angustia, depresión y muerte, hechos que repercuten en ellas y afectan sus expectativas en los ámbitos familiar, laboral y social. Las mujeres manifiestan múltiples preguntas, como las siguientes: ¿cuándo les llegará la hora de morir?, ¿por qué a ellas?, ¿cómo su ausencia influirá en sus hijos?, ¿quién los cuidará en el futuro? También se refleja en ellas el miedo a qué tan doloroso será. Esta situación les recuerda a muchas el abandono y la soledad que vivieron con sus familiares y en especial sus madres. Esto conllevó a identificar la categoría "angustia y depresión por el futuro", de la cual se desprendieron algunos comentarios como los siguientes. 
"Cuando me dijeron que tenía cáncer inmediatamente pensé, me morí, y aunque el médico habló y habló, (...) yo entré en un estado de ausentismo, me deprimí y ya no quería nada" (30R12).

“(...) hace unos años tenía muchos planes, ahora vivo el día a día con angustia de lo que me pueda pasar y más a mis hijitos que se queden solos, sin mamá” (3R32).

En todo proceso patológico es indispensable el apoyo social que tenga la persona. En el caso de las mujeres con cáncer de mama, la situación no es diferente, pues su raíz y fortaleza se la dan sus familias. A pesar de que la enfermedad ha generado en ellas un aislamiento social, consideran que su apoyo y fuerza son sus hijos: por ellos, mencionan que lucharán hasta vencer o morir. De esta manera, sobresale la cuarta categoría: "la familia, un apoyo fundamental", como se evidencia en los siguientes relatos.

“(...) mi familia es mi pilar y mi fortaleza, ellos han estado en los momentos más duros de esto, sino fuera por ellos creo que no le diera la pelea por seguir viva" (27R22).

“(...) mis hijos son el motor para seguir en la lucha, han estado al lado mío dando ánimo, no quiero verlos sufrir y por ellos saco la fuerza para sobrevivir a esta enfermedad, que me desmorona poco a poco" (18R5).

\section{Discusión}

Se pudo identificar que las mujeres presentan una edad promedio de 46,7 años, lo que concuerda con estudios a nivel mundial donde se evidencia que la prevalencia del cáncer de mama relacionada con la edad corresponde a mujeres mayores de 40 años (Talley y Williams, 2015). Estudios infieren y sustentan que la aparición del cáncer de mama está mediado por factores endocrinos relacionados con "la duración de la vida menstrual de la mujer, en especial la menarca, (...), el inicio temprano de la menopausia, la nuliparidad y el tener un hijo después de los 30 años de edad" (Gómez, Altagracia, Kravzov, Cárdenas y Rubio, 2008, p. 62). Por otro lado, "la manifestación tardía de la menopausia (55 años o más) incrementa 2,5 veces el riesgo, mientras que la menopausia artificial por castración quirúrgica, antes de los 40 años, ha demostrado disminución significativamente el riesgo" (Rodríguez y Capurso, 2006, p. 589). Estos hecho concuerdan con lo encontrado en este proceso investigativo, que además resalta la importancia de generar estrategias de prevención hacia la población femenina en edades previas a los 40 años.

El estudio evidenció que gran parte de las mujeres presentan antecedentes familiares asociados al cáncer, y por las evidencias, se ha determinado que puede ser el "responsable de 
la mayoría de los cánceres de mama familiares/hereditarios; estos últimos constituyen del 5\% al 10\% del total de cánceres de mama" (Sanabria et al., 2009. p. 62). En ese mismo sentido, el estudio investigativo de Pinto et al. (2006, p. 7) indicó que los cánceres "son de origen familiar, de los cuales el 5\% se puede asociar con mutaciones en genes de alta penetrancia (sic) como BRCA1 y BRCA2", situación que debe ser una acción a detectar para la prevención de la familia.

Basado en lo anterior, se confirma la categoría "el cáncer como una enfermedad heredada". Además, se explica a partir de que "la expresión génica aberrante es una de las características clave vinculada con enfermedades complejas como cáncer” (García, Ayala y Perdomo, 2012, p. 64), pues la predisposición familiar que tiene el cáncer de mama "es una herencia dominante, con un 50\% de riego de transmitir los alelos de las mutaciones a los hijos (...) generando una mutación de la línea germinal en genes altamente penetrantes tales como el BRC1 y BRC2" (Molina, Villarroel, Arata-Bellabarta y LaCruz, 2008, p. 26).

De la misma manera, se ha establecido que el cáncer, en cualquier manifestación, tiene componentes heredables; por ello, las mujeres ya son conocedoras de todo el proceso que desarrolla y las consecuencias que tiene esta enfermedad, por lo que manifiestan su temor y angustia a heredarles esta enfermedad a sus hijas (Palacios-Espinosa et al., 2015) y pasar lo mismo que vivieron con sus familiares fallecidos. De ello, sobresale que las campañas de prevención hacia familiares de mujeres con cáncer de mama u otros tipos requieren de visiones amplias desde la salud pública tanto para minimizar la tasa de afección como para velar por los factores epigenéticos relacionados con el cáncer.

El trabajo investigativo permitió determinar, en términos generales, que el cáncer de mama tiene en las mujeres repercusiones importantes en su manera de verse, tanto corporal como sexualmente. Se advierte que en patologías mamarias se producirán "efectos psicológicos sobre la persona, a causa de cambios que ocurren en la autoconceptualización y en la imagen corporal" (García Viniegras y González Blanoc, 2007, p. 74), ya que "el cáncer de mama en particular tiene una naturaleza insidiosa porque agrede a un órgano íntimamente asociado con la autoestima, la sexualidad y la femineidad" (Garduño, Riveros y Sánchez-Sosa, 2010, p. 70). A esto se suma un impacto en los sentimientos del atractivo y del deseo sexual, pues se reportan cambios en la disminución de la frecuencias de las relaciones sexuales y disminución del deseo sexual, dificultad para relajarse y disfrutar del sexo, así como dificultad para llegar al orgasmo (Juárez y Landero, 2011). Por esto, es importante que las mujeres con cáncer, en cualquier estadio, tengan un adecuado y oportuno apoyo psicológico para afrontar la situación y mejorar tanto su calidad de vida, como su salud.

Lo anterior se suma a la categoría encontrada en los relatos de "angustia y depresión por el futuro". Esta situación es similar a estudios en los que se indica que "la depresión en pacientes con cáncer es tres veces superior que la de población general y dos veces mayor en personas hospitalizadas" (Bejarano et al., 2009, p. 183). En este sentido, Landa, Cárdenas, Greer, Sánchez y Riveros (2014), en una revisión sistemática, reportaron una prevalencia de hasta $24 \%$ de pacientes con cáncer afectados por depresión. Estos síntomas se deben, en gran 
parte, a que los tratamientos utilizados propician cambios en la rutina diaria, los hábitos y los estilos de vida de las mujeres, lo cual a su vez incide en la percepción de sí mismas y del futuro. Según lo manifiesta Fey (2002, p. 309), "experimentamos angustia de lo que fue y lo que ya no podrá volver a ser", por lo que, ante el cáncer de mama, la mujer se angustia por vislumbrar "la potencial aniquilación de su existencia por causa de una amenaza perdida de sostén apoyo en el mundo" (Längle, 2005, p. 60), situación que expresan como angustia por su futuro. De esto se puede inferir que el apoyo de un grupo interdisciplinario es de suma importancia tanto para la estabilidad clínica como psicológica de la mujer.

Es indudable que el temor por tener cáncer de mama genera situaciones angustiantes y depresivas, por eso el soporte de su primera red de apoyo es indispensable para superar y afrontar la enfermedad. De esta situación, se generó la categoría "la familia, un apoyo fundamental", en donde las mujeres aprecian y ven a su familia como la fortaleza y sostén para sobrellevar el cáncer. Esta apreciación se constata en los estudios Palacios et al. (2015, p. 505), quienes infieren que "de forma prácticamente inmediata, se hará evidente esa red de apoyo familiar cuya compleja dinámica se alimenta de apoyar al paciente $\mathrm{y}$, a la vez, de recibir apoyo entre los diferentes miembros que la componen". De esta manera, se hace evidente que la familia adoptará acciones a esta situación "utilizando mecanismos de enfrentamiento para superar las crisis consecuentes” (Mesquita et al., 2007, p. 3). Es así como se ve a la familia más unida en torno a uno de sus familiares enfermos y emplea diferentes medios para mantener el bienestar de su ser querido. Uno de los medios utilizados es la comida, que representa "el medio más cercano que la familia tiene para ofrecer cuidados, afecto y ayuda a su ser querido que se manifiesta cada vez más inapetente conforme progresa la enfermedad" (Bejarano et al., 2009. p. 183).

Lo planteado fundamenta el proceso investigativo presentado, tanto en el ámbito local, con este grupo de mujeres, como a nivel internacional. De esta manera, se marcan puntos de partida para una atención más personalizada, desde la salud pública, y conducente a asociar otras acciones a favor de las personas con cáncer.

\section{Conclusiones}

Esta investigación pone de manifiesto que el cáncer es una enfermedad de probabilidad heredada y que son los hijos de quienes vivieron el proceso de la enfermedad, en sus mismas familias, los que ven reflejado su padecer en el presente propio. Esta situación indica la necesidad de emplear estrategias de prevención a la familia de quienes presentan cáncer, ya sea por seguimiento continuo -como mayor información desde la atención primaria en salud- o a través de pruebas clínicas continuas desde la base genética para confirmar la presencia de dichos genes.

La información acerca de la percepción que tienen las mujeres sobre el cáncer es importante pues no solo beneficia a la comunidad, sino que también implica mayor vinculación del personal de salud hacia el trabajo mancomunado de disminuir los índices de mortalidad de 
esta enfermedad. Desde este aspecto, se hace relevante la conformación de grupos de apoyo tanto para los pacientes como para la familia, vinculando desde las instituciones prestadoras del servicio de salud un soporte interdisciplinario para esta enfermedad. Esto incide en que tanto centros de atención como hospitales generen campañas publicitarias y vínculos para el conocimiento propio de la enfermedad como para la prevención hacia el cuidado de los factores de riesgo estudiados y así disminuir la incidencia.

El conocimiento de un diagnóstico de cáncer genera en el individuo sentimientos de angustia, desesperanza y depresión, por lo que debe ser apoyado por un equipo interdisciplinario que brinde al paciente herramientas útiles para mejorar y también le brinde a la familia caminos para sobrellevar y afrontar positivamente la situación.

Es evidente que después de un proceso cancerígeno se presenten secuelas en el paciente que se manifiestan como inseguridades sobre su cuerpo y su rol dentro de una relación y dentro de la sociedad. Esta situación amerita estudios más profundos, pero a la vez es necesario proporcionar un acompañamiento, por parte del personal de salud, sobre la necesidad de la aceptación y la construcción de la autoestima en el paciente, lo cual fundamental para afrontar su vida emocional, laboral y social. Con base en lo anterior, se recomienda el trabajo psicosocial, que debe ir acompañando al tratamiento de la enfermedad, de igual manera que el apoyo de su cónyugue, para disminuir el impacto en la relación de pareja.

\section{Agradecimientos.}

A las participantes y amigas de este estudio que permitieron un conocimiento mayor desde sus experiencias vividas, al personal médico y enfermeras del Hospital Universitario de San José, a los estudiantes que colaboraron ayudándonos a contactar a todas y cada una de nuestras participantes. De la misma manera, a mis colegas de la Universidad del Cauca, en especial del Departamento de Educación Física, Recreación y Deporte. Y a los docentes del Doctorado en ciencias Biomédicas de la Universidad del Valle.

Recepción: 6 de mayo del 2016

Corrección: 12 de septiembre del 2016

Aceptación: 24 de octubre del 2016

Publicación: 6 de diciembre del 2016

\section{Referencias bibliográficas}

Aguilar Cordero, M. J., González Jiménez, E., García López, P., Álvarez Ferre, J., \& Padilla López, C. A. (2012). Obesity and seric levels of strogens; the importance of early 
development of breast cancer. Nutrición Hospitalaria, 27(4), 1156-1159. http://doi. org/10.3305/nh.2012.27.4.5854

Baider, L. (2003). Cáncer y familia : aspectos teóricos y terapéuticos. International Journal of Clinical and Health Psychology, 3(3), 505-520. Recuperado de http:/www.redalyc.org/ articulo.oa?id=33730306

Bejarano, M., Fuchs, V., Fernández, N., \& Amancio, O. (2009). Impacto del acompañamiento familiar sobre la ingestión de alimentos y el estado depresivo en pacientes con cáncer cervicouterino hospitalizadas. Nutrición Hopitalaria, 24(2), 182-186. Recuperado de http://www.redalyc.org/articulo.oa?id=30922674400

Chen, Y., Wen, Y., Li, Z., Luo, D., \& Zhang, X. (2016). The molecular mechanisms between metabolic syndrome and breast cancer. Biochemical and Biophysical Research Communications, 12-16. http://doi.org/10.1016/j.bbrc.2016.02.034

Declaración de Helsinki. Principios éticos para las investigaciones médicas en seres humanos (1975). Tokio-Japón: Asociación Medica Mundial.

Fey, E. (2002). Consideraciones sobre la Nada y la Angustia. Signos Filosóficos, 8, 305-311. Recuperado de http://www.redalyc.org/articulo.oa?id=34300818

Figueredo Villa, K. (2008). Ciudados paliativos. Una opción vital para pacientes con cáncer de mama. Revista Habanera de Ciencias Médicas, 7, 1-12. Recuperado de http://www. redalyc.org/articulo.oa?id=180414029008

García Robles, R., Ayala Ramírez, A. P., \& Perdomo Velásquez, S. (2012). Epigenética: definición, bases moleculares e implicaciones en la salud y en la evolución humana. Revista Ciencias de La Salud, 10, 59-71.

García Viniegras, C. R., \& González Blanoc, M. (2007). Bienestar psicológico y cáncer de mama. Avances En Psicología Latinoamericana, 25, 72-80. Recuperado de http://www. redalyc.org/articulo.oa? $\mathrm{id}=79902508$

Garduño, C., Riveros, A., \& Sánchez-Sosa, J. J. (2010). Calidad de Vida y Cáncer de Mama: Efectos de una Intervención Cognitivo-Conductual. Revista Latinoamericana de Medicina Conductual, 1, 69-80. Recuperado de http://www.redalyc.org/articulo.oa?id=283021975008

Gómez, J., Altagracia, M., Kravzov, J., Cárdenas, R., \& Rubio Poo, C. (2008). Cáncer de mama y las actuales alternativas de tratamiento Breast cancer and Present Treatment alternatives. Revista Mexicana de Ciencias Farmacéuticas, 39(3), 58-70. Recuperado de http://redalyc. uaemex.mx/src/inicio/ArtPdfRed.jsp?iCve $=57911110008$

González-Robledo, L. M., González-Robledo, M. C., Nigenda, G., \& López-Carrillo, L. (2010). Acciones gubernamentales para la detección temprana del cáncer de mama en América 
Latina. Retos a futuro. Revista de Salud Pública de México, 52, 533-543. Recuperado de http://www.redalyc.org/articulo.oa?id=10618968008

Guevara-López, U. (2007). El dolor o los dolores. El nuevo paradigma. Cirugía Y Cirujanos, 75(2), 63-64. Recuperado de http://www.redalyc.org/articulo.oa?id=66275201

Hernández Sampieri, R., Fernández Collado, C., \& Baptista Lucio, M. del P. (2014). Metodología de la Investigación. México: McGraw-Hill, Ed.

Juárez García, D., \& Landero Hernández, R. (2011). Imagen corporal , funcionamiento sexual , autoestima y optimismo en mujeres con cáncer de mama. Nova Scientia, 4, 17-34. Recuperado de http://www.redalyc.org/articulo.oa?id=203320117002

Knaul, F. M., López, L., Lazcano, E., Gómez, H., Romieu, I., \& Torres, G. (2009). Cáncer de mama: un reto para la sociedad y los sistemas de salud. Salud Pública de México, 51(2), 138-140. Recuperado de http://www.redalyc.org/articulo.oa?id=10616164002

Landa-Ramírez, E., Cárdenas-López, G., Andrew Greer, J., Sánchez-Román, S., \& RiverosRosas, A. (2014). Evaluación de la depresión en pacientes con cáncer terminal y su aplicación en el contexto mexicano: Una revisión. Salud Mental, 37(5), 415-422. Recuperado de http://www.inprf-cd.gob.mx/pdf/sm3705/sm3705415.pdf

Längle, A. (2005). La Búsqueda de Sostén . Análisis Existencial de la Angustia. Terapia Psicologíca, 23(2), 57-64. Recuperado de http://www.redalyc.org/articulo.oa?id=78523206

Londoño Palacio, O., \& Chaparro Borja, P. (2011). Condiciones de hábitat y percepción de los estados de salud. Conceptos categoriales emergentes. Hacia La Promoción de La Salud, (2), 13-31.

Mesquita Melo, E., Magalhães da Silva, R., De Almeida, A. ., Carvalho Fernández, A. F., \& Mota Rego, C. D. (2007). Comportamiento de la familia frente al diagnóstico de cáncer de mama. Enfermería Global, 6, 1-10.

Ministerio de Salud y Protección Social. Resolución 8430 (1993).

Molina, C., Villarroel, V., Arata-Bellabarta, G., \& LaCruz, J. (2008). Importancia de la historia familiar en el cáncer de mama y ovario. Revista Venezolana de Endocrinología $Y$ Metabolismo, 6, 25-29.

Palacios-Espinosa, A. M., \& Castaño Rodríguez, X. (2013). Representaciones Sociales del Cáncer y de la Quimioterapia. Psicooncología, 10(79-93), 79-93. http://doi.org/10.5209/rev

Palacios-Espinosa, X., Gonzáles, M. I., \& Zani, B. (2015). Las representaciones sociales del cáncer y de la quimioterapia en la familia del paciente oncológico. Avances En Psicología Latinoamericana, 33(3), 1. 
Pinto, Y., Sánchez, W., Ibáñez, M., \& Ramírez, S. (2006). Polimorfismos del gen P53 en Cáncer Mamario familiar en una Población Colombiana. Revista de La Universidad Industrial de Santander. Salud, 38, 7-8.

Pou, S. A., Niclis, C., Aballay, L. R., Tumas, N., Román, M. D., Muñoz, S. E., ... Díaz, M. del P. (2014). Cáncer y su asociación con patrones alimentarios en Córdoba (Argentina). Nutricion Hospitalaria, 29(3), 618-628. http://doi.org/10.3305/nh.2014.29.3.7192

Rodríguez Cuevas, S., \& Capurso García, M. (2006). Epidemiología del cáncer de mama. Ginecol Obstet Mex, 74, 585-593. Recuperado de http:/www.medigraphic.com/pdfs/ ginobsmex/gom-2006/gom0611f.pdf

Sanabria, M. C., Muñoz, G., \& Vargas, C. I. (2009). Análisis de las mutaciones más frecuentes del gen BRCA1 (185delAG y 5382insC) en mujeres con cáncer de mama en Bucaramanga, Colombia. Biomédica Bogot, 29(1), 61-72. Recuperado de http://redalyc.uaemex.mx/ pdf/843/84311628009.pdf

Talley, C. H., \& Williams, K. P. (2015). Impact of Age and Comorbidity on Cervical and Breast Cancer Literacy of African Americans, Latina, and Arab Women. Nurs Clin N Am, 50, 545-563. Recuperado de http://dx.doi.org/10.1016/j.cnur.2015.05.008

Valle Rivadeneyra, R. E., Zúñiga, M., Tuzet, C., Martínez, C., De la Jara, J., Aliaga, R., \& Whittembury, Ä. (2006). Sintomatología depresiva y calidad de vida en pacientes mujeres con cáncer de mama. Anales de La Facultad de Medicina, 67(4), 327-332. Recuperado de http://www.redalyc.org/articulo.oa?id=37967407

Varela Arévalo, M. T., Arrivillaga Quintero, M., Cáceres, D. elena, Correa Sánchez, D., \& Holguin Palacios, L. E. (2005). Efectos benéficos de la modificación del estilo de vida en la presión arterial y la calidad de vida en pacientes con hipertensión. Acta Colombiana de Psicología, 14, 69-85.

\footnotetext{
Nancy Janneth Molano-Tobar. Fisioterapeuta, Magister en Educación, Candidata al título de Doctorado en Ciencias Biomédicas de la Universidad del Valle. Docente Titular de la Universidad del Cauca

2 Patricia Eugenia Vélez Varela. Bióloga, Doctora en Ciencias Biomédicas. Docente Titular de la Universidad del Cauca.
} 